\title{
Karel Segeth
}

A particular smooth interpolation that generates splines

In: Jan Chleboun and Pavel Kůs and Petr Přikryl and Karel Segeth and Jakub Šístek and Tomáš Vejchodský (eds.): Programs and Algorithms of Numerical Mathematics, Proceedings of Seminar. Janov nad Nisou, June 19-24, 2016. Institute of Mathematics CAS, Prague, 2017. pp. 112-119.

Persistent URL: http://dml.cz/dmlcz/703005

\section{Terms of use:}

(C) Institute of Mathematics CAS, 2017

Institute of Mathematics of the Czech Academy of Sciences provides access to digitized documents strictly for personal use. Each copy of any part of this document must contain these Terms of use.




Programs and Algorithms of Numerical Mathematics 18

J. Chleboun, P. Kůs, P. Přikryl, K. Segeth, J. Šístek, T. Vejchodský (Eds.)

Institute of Mathematics CAS, Prague 2017

\title{
A PARTICULAR SMOOTH INTERPOLATION THAT GENERATES SPLINES
}

\author{
Karel Segeth \\ Institute of Mathematics, Czech Academy of Sciences \\ Žitná 25, CZ-115 67 Prague 1, Czech Republic \\ segeth@math.cas.cz
}

\begin{abstract}
There are two grounds the spline theory stems from - the algebraic one (where splines are understood as piecewise smooth functions satisfying some continuity conditions) and the variational one (where splines are obtained via minimization of some quadratic functionals with constraints). We use the general variational approach called smooth interpolation introduced by Talmi and Gilat and show that it covers not only the cubic spline and its 2D and 3D analogues but also the well known tension spline (called also spline with tension). We present the results of a 1D numerical example that characterize some properties of the tension spline.
\end{abstract}

Keywords: data interpolation, smooth interpolation, spline interpolation, tension spline, Fourier series, Fourier transform

MSC: 65D05, 65D07, 41A05, 41A63, 42A38

\section{Introduction}

The minimum curvature approach to interpolation, which produces e.g. cubic splines, usually provides a visually nice smooth curve or surface. However, in some cases the method can create artificial oscillations. A remedy proposed by Schweikert [4] is known as tension spline. The functional minimized includes the first derivative term in addition to the second derivative term.

The smooth approximation [8] is an approach to data interpolating or data fitting that employs the variational formulation of the problem in a normed space with constraints representing the approximation conditions. The cubic spline interpolation in $1 \mathrm{D}$ is also known to be the approximation of this kind.

For the cubic spline, the objective is to minimize the $L^{2}$ norm of the second derivative of the approximating function. A more complex criterion then is to minimize, with some weights chosen, the $L^{2}$ norms of several (or possibly all) derivatives of a sufficiently smooth approximating function. In the paper, we are concerned with the tension spline constructed by means of the smooth approximation theory

DOI: $10.21136 /$ panm.2016.14 
(cf. also [3]), i.e. with the exact interpolation of the data at nodes and, at the same time, with the smoothness of the interpolating curve and its first derivative.

For the sake of simplicity, we are mostly concerned with the 1D case in the paper. Assuming the approach of [8] and [5], we introduce the problem to be solved and the tools necessary to this aim in Sec. 3. We also present the general existence theorem for smooth interpolation proven in [5]. We use the basis system $\exp (\mathrm{i} k x)$ of exponential functions of pure imaginary argument for smooth interpolation problems in Sec. 4. In Sec. 5 we investigate some properties of this basis suitable for preserving the smoothness of the interpolation and for generating the tension spline in $1 \mathrm{D}, 2 \mathrm{D}$, and 3D. We also present a 1D numerical example and discuss it to illustrate some properties of smooth interpolation in Sec. 6.

\section{Problem of data interpolation}

Basic notation and fundamental statements are presented, e.g., in [6]. Let us have a finite number $N$ of (complex, in general) measured (sampled) values $f_{1}, f_{2}, \ldots, f_{N} \in C$ obtained at $N$ nodes $X_{1}, X_{2}, \ldots, X_{N} \in R^{n}$. The nodes are assumed to be mutually distinct. We are usually interested also in the intermediate values corresponding to other points in some domain. Assume that $f_{j}=f\left(X_{j}\right)$ are measured values of some continuous function $f$ while $z$ is an approximating function to be constructed. The dimension $n$ of the independent variable can be arbitrary.

Definition 1 (Interpolation). The interpolating function (interpolant) $z$ is constructed to fulfil the interpolation conditions

$$
z\left(X_{j}\right)=f_{j}, \quad j=1, \ldots, N .
$$

Various additional conditions can be considered, e.g. minimization of some functionals applied to $z$.

The problem of data interpolation does not have a unique solution. The property (1) of the interpolating function is uniquely formulated by mathematical means but there are also requirements on the subjective perception of the behavior of the approximating curve or surface between nodes that can hardly be formalized.

The general problem of smooth approximation (smooth curve fitting, data smoothing), where the interpolation condition (1) is not applied, is treated in more detail e.g. in [5], [8].

For the sake of simplicity we now put $n=1$ and assume that $X_{1}, X_{2}, \ldots, X_{N} \in \Omega$, where either $\Omega=[a, b]$ is a finite interval or $\Omega=(-\infty, \infty)$. We will turn back to general $n \geq 1$ in Sec. 5 .

\section{Smooth interpolation}

We introduce an inner product space to formulate the additional constraints in the problem of smooth approximation [6], [8]. Let $\widetilde{\mathcal{W}}$ be a linear vector space of complex valued functions $g$ continuous together with their derivatives of all orders 
on the interval $\Omega$. Let $\left\{B_{l}\right\}_{l=0}^{\infty}$ be a sequence of nonnegative numbers and $L$ the smallest nonnegative integer such that $B_{L}>0$ while $B_{l}=0$ for $l<L$. For $g, h \in \widetilde{\mathcal{W}}$, put

$$
\begin{aligned}
(g, h)_{L} & =\sum_{l=0}^{\infty} B_{l} \int_{\Omega} g^{(l)}(x)\left[h^{(l)}(x)\right]^{*} \mathrm{~d} x \\
|g|_{L}^{2} & =\sum_{l=0}^{\infty} B_{l} \int_{\Omega}\left|g^{(l)}(x)\right|^{2} \mathrm{~d} x
\end{aligned}
$$

where ${ }^{*}$ denotes the complex conjugate.

If $L=0$ (i.e. $B_{0}>0$ ), consider functions $g \in \widetilde{\mathcal{W}}$ such that the value of $|g|_{0}$ exists and is finite. Then $(g, h)_{0}=(g, h)$ has the properties of inner product and the expression $|g|_{0}=\|g\|$ is norm in a normed space $W_{0}=\widetilde{\mathcal{W}}$.

Let $L>0$. Consider again functions $g \in \widetilde{\mathcal{W}}$ such that the value of $|g|_{L}$ exists and is finite. Let $P_{L-1} \subset \widetilde{\mathcal{W}}$ be the subspace whose basis $\left\{\varphi_{p}\right\}$ consists of monomials

$$
\varphi_{p}(x)=x^{p-1}, \quad p=1, \ldots, L .
$$

Then $\left(\varphi_{p}, \varphi_{q}\right)_{L}=0$ and $\left|\varphi_{p}\right|_{L}=0$ for $p, q=1, \ldots, L$. Using (2) and (3), we construct the quotient space $\widetilde{\mathcal{W}} / P_{L-1}$ whose zero class is the subspace $P_{L-1}$. Finally, considering $(\cdot, \cdot)_{L}$ and $|\cdot|_{L}$ in every equivalence class, we see that they represent the inner product and norm in a normed space $W_{L}=\widetilde{\mathcal{W}} / P_{L-1}[6]$.

$W_{L}$ is the normed space where we minimize functionals and measure the smoothness of the interpolation. For an arbitrary $L \geq 0$, choose a basis system of functions $\left\{g_{k}\right\} \subset W_{L}, k=1,2, \ldots$, that is complete and orthogonal (in the inner product in $\left.W_{L}\right)$, i.e., $\left(g_{k}, g_{m}\right)_{L}=0$ for $k \neq m,\left(g_{k}, g_{k}\right)_{L}=\left|g_{k}\right|_{L}^{2}>0$. If $L>0$ then it is, moreover, $\left(\varphi_{p}, g_{k}\right)_{L}=0$ for $p=1, \ldots, L, k=1,2, \ldots$ The set $\left\{\varphi_{p}\right\}$ is empty for $L=0$.

Definition 2 (Smooth data interpolation). The problem of smooth data interpolation [8] consists in finding the coefficients $A_{k}$ and $a_{p}$ of the interpolant

$$
z(x)=\sum_{k=1}^{\infty} A_{k} g_{k}(x)+\sum_{p=1}^{L} a_{p} \varphi_{p}(x)
$$

such that

$$
z\left(X_{j}\right)=f_{j}, \quad j=1, \ldots, N
$$

and

$$
\text { the quantity }|z|_{L}^{2} \text { attains its minimum. }
$$

Apparently, the infinite sum in (4) is inconvenient for practical computation. Therefore, we introduce the generating function

$$
R_{L}(x, y)=\sum_{k=1}^{\infty} \frac{g_{k}(x) g_{k}^{*}(y)}{\left|g_{k}\right|_{L}^{2}} .
$$


We state in Theorem 1, which follows, that a finite linear combination of the values of the generating function $R_{L}$ at particular nodes is used for the practical interpolation instead of the infinite linear combination in (4). Further put

$$
R=\left[R_{L}\left(X_{i}, X_{j}\right)\right], \quad i, j=1, \ldots, N,
$$

where $R$ is an $N \times N$ square Hermitian matrix, and if $L>0$ then introduce an $N \times L$ matrix

$$
\Phi=\left[\varphi_{p}\left(X_{j}\right)\right], \quad j=1, \ldots, N, p=1, \ldots, L .
$$

Theorem 1. Let $X_{i} \neq X_{j}$ for all $i \neq j$. Assume that the generating function (7) converges for all $x, y \in \Omega$. If $L>0$ let rank $\Phi=L$. Then the problem of smooth interpolation (4) to (6) has the unique solution

$$
z(x)=\sum_{j=1}^{N} \lambda_{j} R_{L}\left(x, X_{j}\right)+\sum_{p=1}^{L} a_{p} \varphi_{p}(x),
$$

where the coefficients $\lambda_{j}, j=1, \ldots, N$, and $a_{p}, p=1, \ldots, L$, are the unique solution of a nonsingular system of $N+L$ linear algebraic equations.

Proof. The proof is given in [5].

\section{A particular basis function system}

Recall that we have put $n=1$. Let the function $f$ to be approximated be $2 \pi$-periodic in $[0,2 \pi]$. We choose exponential functions of pure imaginary argument for the periodic basis system $\left\{g_{k}\right\}$ in $W_{L}$. The following theorem shows important properties of the system.

Theorem 2. Let there be an integer $s, s \geq L$, such that $B_{l}=0$ for all $l>s$ in $W_{L}$. The system of periodic exponential functions of pure imaginary argument

$$
g_{k}(x)=\exp (-\mathrm{i} k x), \quad x \in[0,2 \pi], k=0, \pm 1, \pm 2, \ldots,
$$

is complete and orthogonal in $W_{L}$.

Proof. The proof is given in [6].

The range of $k$ implies a minor change in the notation introduced above. For the basis system (9), notice that the generating function

$$
R_{L}(x, y)=\sum_{k=-\infty}^{\infty} \frac{g_{k}(x) g_{k}^{*}(y)}{\left|g_{k}\right|_{L}^{2}}=\sum_{k=-\infty}^{\infty} \frac{\exp (-\mathrm{i} k(x-y))}{\left|g_{k}\right|_{L}^{2}}
$$

is the Fourier series in $L^{2}(0,2 \pi)$ with the coefficients $\left|g_{k}\right|_{L}^{-2}$, where

$$
\left|g_{k}\right|_{L}^{2}=2 \pi \sum_{l=L}^{\infty} B_{l} k^{2 l}
$$

according to (3). 
Let now the function $f$ to be approximated be nonperiodic on $(-\infty, \infty)$ and $f^{(l)}( \pm \infty)=0$ for all $l \geq 0$. Let us define the generating function $R_{L}(x, y)$ as the Fourier transform of the function $\left|g_{k}\right|_{L}^{-2}$ of continuous variable $k$,

$$
R_{L}(x, y)=\int_{-\infty}^{\infty} \frac{\exp (-\mathrm{i} k(x-y))}{\left|g_{k}\right|_{L}^{2}} \mathrm{~d} k
$$

if the integral exists. Using the effect of transition from the Fourier series (10) with the coefficients $\left|g_{k}\right|_{L}^{-2}$ to the Fourier transform (12) of the function $\left|g_{k}\right|_{L}^{-2}$ of continuous variable $k$ (cf., e.g., [6]), we have transformed the basis functions, enriched their spectrum, and released the requirement of periodicity of $f$. Moreover, if the integral (12) does not exist in the usual sense, in many instances we can calculate $R_{L}(x, y)$ as the Fourier transform $\mathcal{F}$ of the generalized function $\left|g_{k}\right|_{L}^{-2}$ of $k$.

\section{Tension spline}

To finish the definition of the inner product and norm (2), (3) in a particular space $W_{L}$ we now choose a particular sequence $\left\{B_{l}\right\}$ and set, therefore, the minimization properties of the smooth interpolant. Let us thus put (cf. [3])

$$
B_{l}=0 \text { for all } l \text { with the exception of } B_{1}=\alpha^{2}, \alpha>0 \text {, and } B_{2}=1 \text {. }
$$

It means that we have $L=1$ and minimize the $L^{2}$ norm of the first derivative (characterizing oscillations) multiplied by $\alpha^{2}$ plus the $L^{2}$ norm of the second derivative (characterizing the curvature) of the interpolant (4) in the form (8), i.e.

$$
z(x)=\sum_{j=1}^{N} \lambda_{j} R_{1}\left(x, X_{j}\right)+a_{1} .
$$

We get

$$
\left|g_{k}\right|_{1}^{2}=2 \pi\left(\alpha^{2} k^{2}+k^{4}\right)
$$

from (11). Let $r=|x-y|$. We arrive at

$$
\begin{aligned}
R_{1}(x, y) & =\mathcal{F}\left(\frac{1}{2 \pi k^{2}\left(\alpha^{2}+k^{2}\right)}\right)=\frac{1}{2 \pi} \mathcal{F}\left(\frac{1}{\alpha^{2} k^{2}}-\frac{1}{\alpha^{2}\left(k^{2}+\alpha^{2}\right)}\right) \\
& =-\frac{1}{2 \alpha^{3}}(\alpha r+\exp (-\alpha r)),
\end{aligned}
$$

where $\mathcal{F}$ denotes the Fourier transform of a generalized function (see [2], p. 375, formula 14 and p. 377, formula 29; and [1], formula 8.469.3), cf. [7]. We see that this version of smooth approximation is equivalent to the tension spline interpolation [4] but introduced in a way different from [3].

For dimension $n>1, l$ is a multiindex, $k, x, y$ are vectors, and the formula (14) remains the same. 
If $n=2$ then $\left|g_{k}\right|_{1}^{2}=4 \pi^{2}\left(\alpha^{2}\left(k_{1}^{2}+k_{2}^{2}\right)+\left(k_{1}^{2}+k_{2}^{2}\right)^{2}\right)$ and we arrive at

$$
\begin{aligned}
R_{1}(x, y) & =\mathcal{F}\left(\frac{1}{4 \pi^{2}\left(\alpha^{2}\left(k_{1}^{2}+k_{2}^{2}\right)+\left(k_{1}^{2}+k_{2}^{2}\right)^{2}\right)}\right) \\
& =-\frac{1}{2 \pi \alpha^{2}}\left(\ln \left(\frac{1}{2} \alpha r\right)-\mathrm{K}_{0}(\alpha r)+C\right),
\end{aligned}
$$

where $C$ is a constant that can be included into $a_{1}$ in (14) and $\mathrm{K}_{0}$ is the modified Bessel function of the second kind (see [2], p. 382, formula 13 and p. 380, formula 5).

Moreover, if $n=3$ then $\left|g_{k}\right|_{1}^{2}=8 \pi^{3}\left(\alpha^{2}\left(k_{1}^{2}+k_{2}^{2}+k_{3}^{2}\right)+\left(k_{1}^{2}+k_{2}^{2}+k_{3}^{2}\right)^{2}\right)$. We finally have

$$
\begin{aligned}
R_{1}(x, y) & =\mathcal{F}\left(\frac{1}{8 \pi^{3}\left(\alpha^{2}\left(k_{1}^{2}+k_{2}^{2}+k_{3}^{2}\right)+\left(k_{1}^{2}+k_{2}^{2}+k_{3}^{2}\right)^{2}\right)}\right) \\
& =\frac{1}{4 \pi \alpha^{2}} \frac{1-\exp (-\alpha r)}{r}
\end{aligned}
$$

(see [2], p. 382, formula 13 and p. 380, formula 1).

At $r=0$, the above functions $R_{1}(x, y)$ are defined as a limit for $r \rightarrow 0$.

There are further practical examples of smooth interpolation where the integral (12) that defines the generating function can be calculated with the help of the Fourier transform. It is easy to show that one of them is the $1 \mathrm{D}$ cubic spline interpolation and its analogues in 2D and 3D [6].

\section{Computational comparison}

We present results of a simple numerical experiment with the tension spline for $n=1$. We employ the complete and orthogonal system (9) and the sequence (13) to introduce the space $W_{1}$. We use the interpolant (14), where $R_{1}$ is given by (15). The function to be interpolated is

$$
f(x)=8-\frac{2}{1+16 x^{2}} .
$$

Apparently, it has "almost a negative pole" at $x=0$. The tension spline interpolation of the function (16) has been constructed in several equidistant grids of $N$ nodes on $[-1,1]$ and for several values of $\alpha^{2}$ including also $\alpha^{2}=0$, i.e. the cubic spline.

Some of the results of interpolation are in Fig. 1. We put $N=9$ and compare tension splines with $\alpha^{2}=0, \alpha^{2}=1000$, and $\alpha^{2}=10000$. The interpolants are in the upper part of the figure, their first derivatives in the lower part along the $x$ axis.

We see that the tension splines do not differ substantially from each other but their derivatives are very unlike. The derivative of the cubic spline is a smooth function while the derivative of the tension spline with $\alpha^{2}=10000$ is similar to a piecewise constant function with smooth changes between the constant levels. This corresponds to the behavior of the tension spline if examined in a different scale: it 


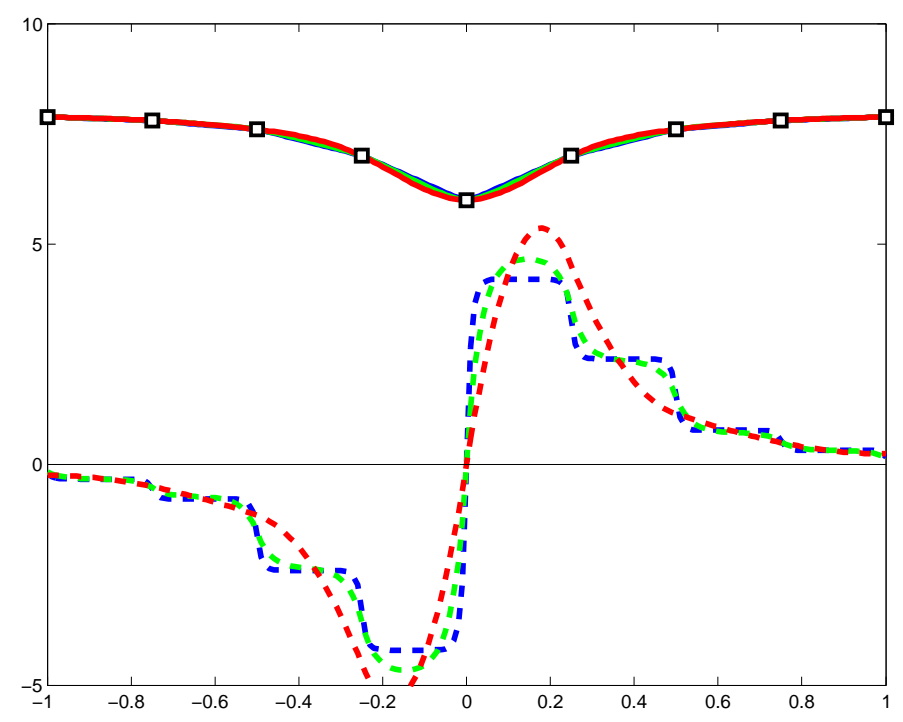

Figure 1: $\quad N=9$. The horizontal axis: independent variable, the vertical axis: interpolant (in the upper part of the figure) and its derivative (in the lower part). Cubic spline (tension $\left.\alpha^{2}=0\right)$ : red line, tension spline $\left(\alpha^{2}=1000\right)$ : green line, tension spline $\left(\alpha^{2}=10000\right)$ : blue line.

resembles a piecewise linear curve but it is smooth, not sharp-cornered also at nodes, i.e. its derivative is continuous.

A proper choice of the parameter $\alpha^{2}$ can provide a compromise interpolation solution with both tension spline and its derivative so smooth that they give a good, pleasing subjective impression.

\section{Conclusion}

We have shown that the generating function for the tension spline interpolation can be obtained by means of the Fourier transform of generalized functions. To this end we have employed the integral definition (12) of the generating function and some known formulae for the Fourier transform. The Fourier transform can be successfully used to determine the generating function also in several other cases including $n=2$ and $n=3$. Moreover, the same approach can be applied to smooth approximation where no interpolation conditions (1) are prescribed. The example in Fig. 1 is a very simple illustration of the $1 \mathrm{D}$ smooth interpolation case.

\section{Acknowledgements}

This work has been supported by the Czech Science Foundation through Grant 14-02067S and by project RVO 67985840. 


\section{References}

[1] Gradshteyn, I. S. and Ryzhik, I. M.: Table of integrals, series, and products. Academic Press, Boston, 1994.

[2] Krě̌n, S. G. (Ed.): Functional analysis (Russian). Nauka, Moskva, 1964.

[3] Mitáš, L. and Mitášová, H.: General variational approach to the interpolation problem. Comput. Math. Appl. 16 (1988), 983-992.

[4] Schweikert, D. G.: An interpolation curve using a spline in tension. J. Math. Phys. 45 (1966), 312-317.

[5] Segeth, K.: Some computational aspects of smooth approximation. Computing 95 (2013), S695-S708.

[6] Segeth, K.: A periodic basis system of the smooth approximation space. Appl. Math. Comput. 267 (2015), 436-444.

[7] Segeth, K.: A note on tension spline. In: Applications of Mathematics 2015, pp. 217-224. Institute of Mathematics CAS, Prague, 2015.

[8] Talmi, A. and Gilat, G.: Method for smooth approximation of data. J. Comput. Phys. 23 (1977), 93-123. 